\title{
Sudden Unexplained Infant Death
}

National Cancer Institute

\section{Source}

National Cancer Institute. Sudden Unexplained Infant Death. NCI Thesaurus. Code

C111853.

Unexpected death of an infant less than one year of age, whose cause of death is not immediately obvious prior to investig ation. 